\title{
Consensus report from the 7th International Forum for Liver Magnetic Resonance Imaging
}

\author{
Elmar M. Merkle ${ }^{1}$. Christoph J. Zech ${ }^{1}$. Carlo Bartolozzi ${ }^{2}$ - Mustafa R. Bashir ${ }^{3}$. \\ Ahmed Ba-Ssalamah ${ }^{4}$ • Alexander Huppertz ${ }^{5}$. Jeong Min Lee ${ }^{6}$. Jens Ricke ${ }^{7}$. \\ Michiie Sakamoto $^{8}$ - Claude B. Sirlin ${ }^{9} \cdot$ Sheng-Long Ye ${ }^{10} \cdot$ Mengsu Zeng $^{11}$
}

Received: 11 March 2015 /Revised: 21 May 2015 / Accepted: 2 June 2015 / Published online: 13 June 2015

(C) The Author(s) 2015. This article is published with open access at Springerlink.com

\begin{abstract}
Objectives Liver-specific MRI is a fast-growing field, with technological and protocol advancements providing more robust imaging and allowing a greater depth of information per examination. This article reports the evidence for, and expert thinking on, current challenges in liver-specific MRI, as discussed at the 7th International Forum for Liver MRI, which was held in Shanghai, China, in October 2013.

Methods Topics discussed included the role of gadoxetic acid-enhanced MRI in the differentiation of focal nodular hyperplasia from hepatocellular adenoma and small hepatocellular carcinoma (HCC) from small intrahepatic cholangiocarcinoma (in patients with chronic liver disease), the differentiation of low-grade dysplastic nodule (DN) from pre-malignant high-grade DN and early $\mathrm{HCC}$, and treatment planning and assessment of treatment response for patients with $\mathrm{HCC}$ and colorectal liver metastasis. Optimization of the gadoxetic acid-enhanced MRI protocol to gain robust arterial and hepatobiliary phase images was also discussed.
\end{abstract}

Elmar M. Merkle

elmar.merkle@usb.ch

1 Clinic of Radiology and Nuclear Medicine, University Hospital Basel, Petersgraben 4, CH-4031 Basel, Switzerland

2 Department of Radiology, University of Pisa, Pisa, Italy

3 Department of Radiology, Duke University Medical Center, Durham, NC, USA

4 Department of General and Pediatric Radiology, Medical University of Vienna, Vienna, Austria

5 Imaging Science Institute Charité, Berlin, Germany
Results and conclusions Gadoxetic acid-enhanced MRI demonstrates added value for the detection and characterization of focal liver lesions and shows promise in a number of new indications, including regional liver functional assessment and patient monitoring after therapy; however, more data are needed in some areas, and further developments are needed to translate cutting-edge techniques into clinical practice.

Key Points

- Liver-specific MRI is a fast-growing field, with many technological and protocol advancements.

- Gadoxetic acid-enhanced MRI demonstrates value for detecting and characterizing focal liver lesions.

- Gadoxetic acid-enhanced MRI shows promise in regional functional assessment and patient monitoring.

- Further developments are needed to translate cutting-edge techniques into clinical practice.

Keywords Gadoxetic acid $\cdot$ Hepatocellular carcinoma $\cdot$ Liver function tests $\cdot$ Liver neoplasms $\cdot$ Magnetic resonance imaging

6 Department of Radiology, Seoul National University Hospital, Seoul, South Korea

7 Department of Radiology and Nuclear Medicine, Otto-von-Guericke Universität Magdeburg, Magdeburg, Germany

8 Department of Pathology, Keio University School of Medicine, Tokyo, Japan

9 Liver Imaging Group, Department of Radiology, University of California, San Diego, California, USA

10 Department of Hepatic Oncology, Zhongshan Hospital, Fudan University, Shanghai, People's Republic of China

11 Radiologic Diagnostic Department, Zhongshan Hospital, Fudan University, Shanghai, People's Republic of China 


\section{Introduction}

There is growing evidence for the benefits of gadoxeticacid-enhanced magnetic resonance imaging (MRI) in a number of indications for liver imaging. Topics in this area were discussed by more than 70 international abdominal experts at the 7th International Forum for Liver MRI, held in Shanghai, China, in October 2013. Forum participants attended presentations by the authors of this article on the following related topics and discussed these data further in four workgroups:

1. Liver lesion characterization - challenging differential diagnoses

2. Differentiation of low-grade dysplastic nodule (DN) from pre-malignant high-grade DN and early hepatocellular carcinoma (HCC)

3. The role of Primovist ${ }^{\circledR}$ in treatment planning and assessment of treatment response

4. Tips and tricks session - how to improve imaging with Primovist ${ }^{\circledR}$ - differences versus traditional agents

Consensus statements on each topic were proposed based on available evidence and expert opinions of participants. The whole forum further discussed, amended, and voted on the statements, using an electronic voting system with the options 'agree', 'disagree' or 'abstain'. The final consensus statements and the voting results are presented in this article, with the intent to inform radiologists and assist them in their daily clinical practice.

\section{Challenging differential diagnoses in liver lesion characterization}

\section{Focal nodular hyperplasia (FNH) and hepatocellular adenoma (HCA)}

FNH and HCA are benign hepatocellular tumours; differentiating is important because FNHs are usually managed conservatively, while HCAs are managed according to risk of spontaneous bleeding and malignant transformation [1]. Distinguishing between FNH and HCA using dynamic contrast-enhanced (CE) computed tomography (CT) or MRI can be challenging when hallmark features (e.g. central scar) are absent, due to overlapping imaging features.

Consensus statement 1.1 The hepatobiliary phase (HBP) of gadoxetic acid-enhanced MRI can help in the differential diagnosis of FNH and HCA. Specifically, hyperintensity or isointensity relative to the liver, which may be diffuse or peripheral, favours FNH. Delineation of a hypointense central scar strongly favours FNH. Diffuse hypointensity relative to the liver strongly favours HCA. [67/69 (97.1\%) agreement]

The HBP imaging features of FNH on gadoxetic acidenhanced MRI commonly include iso- or hyperenhancement in the HBP (largely strong homogeneous enhancement) [1-4], with hypoenhancement of any central scar relative to the rest of the lesion on T2-weighted images [1]. On the other hand, for HCA, hypointensity in the HBP, relative to the surrounding liver parenchyma, is common $[2,5,6]$. Recent evidence suggests that the HBP of gadoxetic acid-enhanced MRI has a sensitivity and positive predictive value (PPV) of $96 \%$ for differentiation of FNH and HCA [7], and may increase correct lesion diagnosis and reader confidence versus unenhanced and dynamic images alone [8].

Consensus statement 1.2 Further research is needed to determine the role of the HBP of gadoxetic acid-enhanced MRI in predicting HCA subtype. [71/73 (97.3\%) agreement]

Only a few studies $[5,9,10]$ are currently available to assess the value of HBP images from gadoxetic-acidenhanced MRI in differentiating HCA subtypes; however, uptake in the HBP appears to be useful additional information.

\section{Small intrahepatic cholangiocarcinoma (ICC) and HCC in patients with chronic liver disease}

Patients with liver cirrhosis or chronic hepatitis are at risk of developing malignant liver lesions - most commonly HCC, but also ICC. Differential diagnosis is important, as prognoses and patient management differ $[11,12]$. Using extracellular agents, portal venous and delayed-phase images help in the differentiation; progressive or sustained concentric enhancement favours ICC, whereas diffuse or nodular washout appearance favours HCC. Using gadoxetic acid, the differential diagnosis is more difficult as both lesions typically are hypointense in the hepatobiliary phase. Therefore, recent retrospective studies have attempted to identify discriminatory imaging features using gadoxetic acid.

Consensus statement 1.3 In patients at increased risk for HCC with small nodules $(<3 \mathrm{~cm})$ that are suspicious for malignancy but lack hallmark imaging features of HCC, reliable differentiation between HCC and ICC may not be possible.

Gadoxetic acid-enhanced MRI features that favour ICC over HCC include:

- lobulated shape

- rim enhancement in the arterial phase

- target appearance on diffusion-weighted images

- target appearance in the HBP 
Gadoxetic acid-enhanced MRI features that favour HCC over ICC include:

- intralesional fat

- diffuse hyperintensity on pre-contrast T1-weighted imaging

- nodule-in-nodule architecture

- diffuse hyperintensity relative to liver in the HBP

\section{[63/72 (87.5\%) agreement]}

Consensus statement 1.4 Prospective studies in consecutive patients are needed to refine and validate gadoxetic acidenhanced MRI features for differentiation of small ICC and $\mathrm{HCC}$ in patients at increased risk for HCC. [62/72 (87.5\%) agreement]

Univariate analyses of imaging features in gadoxetic-acidenhanced MRI that were predictive of ICC over HCC revealed significance $(P \leq 0.005)$ for lobulated shape, arterial phase rim enhancement, target appearance in the HBP (central hyperintensity with a peripheral hypointense rim on the 10- or 20-min images) and target diffusion-weighted imaging (DWI) appearance (central hypointensity and peripheral hyperintensity on $\mathrm{b}=800 \mathrm{~s} / \mathrm{mm}^{2}$ images) (Fig. 1); however, only target appearance on DWI remained significant in multivariate analyses [13]. Other studies reported lobulated shape, arterial phase rim enhancement and target HBP appearance in varying proportions of ICCs, thus these features are suggestive, but not conclusive, of ICC [14-19].

Although uncommon, imaging features on gadoxetic-acid-enhanced MRI that are suggestive of $\mathrm{HCC}$ in patients with chronic liver disease include diffuse hyperintensity in the HBP, diffuse hyperintensity on pre-contrast T1-weighted images, presence of intralesional fat and a nodule-in-nodule appearance [20, 21]. To our knowledge, these MRI features have not been described for ICC before. Furthermore, intralesional fat and nodule-in-nodule appearance are not included in the WHO's description of ICC pathology [22].

\section{Differentiation of low-grade DN from pre-malignant high-grade DN and early HCC}

Detection of pre-malignant nodules and early HCCs in patients with chronic liver disease improves the chances of curative treatment as progression after radiofrequency ablation (RFA) is less frequent in hypovascular versus hypervascular small HCC $[23,24]$. On CE-MRI, these pre-malignant nodules often lack the hallmark imaging features of $\mathrm{HCC}$ and pose a diagnostic challenge.
Consensus statement 2.1 Hypointensity in the HBP of gadoxetic acid-enhanced MRI provides high sensitivity for the detection of iso-/hypovascular pre-malignant (HGDN) and malignant (eHCC) hepatocellular lesions; however, differentiation of these lesions is not currently possible. [67/73 $(91.8 \%$ agreement]

Consensus statement 2.2 Gadoxetic acid-enhanced MRI, incorporating a combination of ancillary features (lesion size, lesion growth, T2 intensity, DWI, T1 intensity, HBP intensity and intralesional fat), improves risk stratification of patients with chronic liver disease and lesions without hallmark vascular features of HCC. [51/57 (89.5\%) agreement]

Progression of pre-malignant/early HCC has been linked to size $(>1 \mathrm{~cm})$ [25-30], and an increased risk of hypervascularization is associated with the presence of fat $(P<0.01)$, enlargement during follow-up $(P=0.04)$, T2 hyperintensity $(P=0.06)[29,31]$ and hyperintensity on DWI [31].

Hypointensity in the HBP of gadoxetic acid-enhanced MRI is suggestive of pre-malignancy or malignancy (irrespective of lesion vascularity) and can increase the sensitivity of diagnosis [32], with almost all HCC and some high-grade DNs being hypointense in this phase [33]. While some low-grade DNs and regenerative nodules (RNs) also show hypointensity in the HBP [34, 35], these nodules are usually $<1 \mathrm{~cm}$ [36], while high-grade DN and early HCC tend to be $>1 \mathrm{~cm}$. Enhancement ratios for gadoxetic acid decreased with nodule differentiation, but are unable to distinguish between DN and hypovascular well differentiated HCC due to considerable overlap [35, 37].

\section{The role of gadoxetic acid-enhanced MRI in treatment planning and assessment of treatment response}

\section{Pre-operative diagnosis and staging of liver lesions}

Wider use of local treatments and more aggressive surgical options require more meticulous pre-operative planning, alongside volume-based functional analyses [38, 39]. Gadoxetic acid-enhanced MRI can provide comprehensive morphological and regional functional information in a single examination and is showing promise as a pre-operative assessment tool.

\section{Colorectal liver metastases (CRLM)}

Hepatic metastases occur in more than half of patients with primary colorectal cancer [40]. Around 20-25\% of patients with CRLM will present with resectable disease [40, 41]. Patient selection and therapy planning may be improved by 
Fig. 1 Target appearance of intrahepatic cholangiocarcinoma on gadoxetic acid-enhanced MRI and diffusion-weighted imaging. Images courtesy of Claude Sirlin

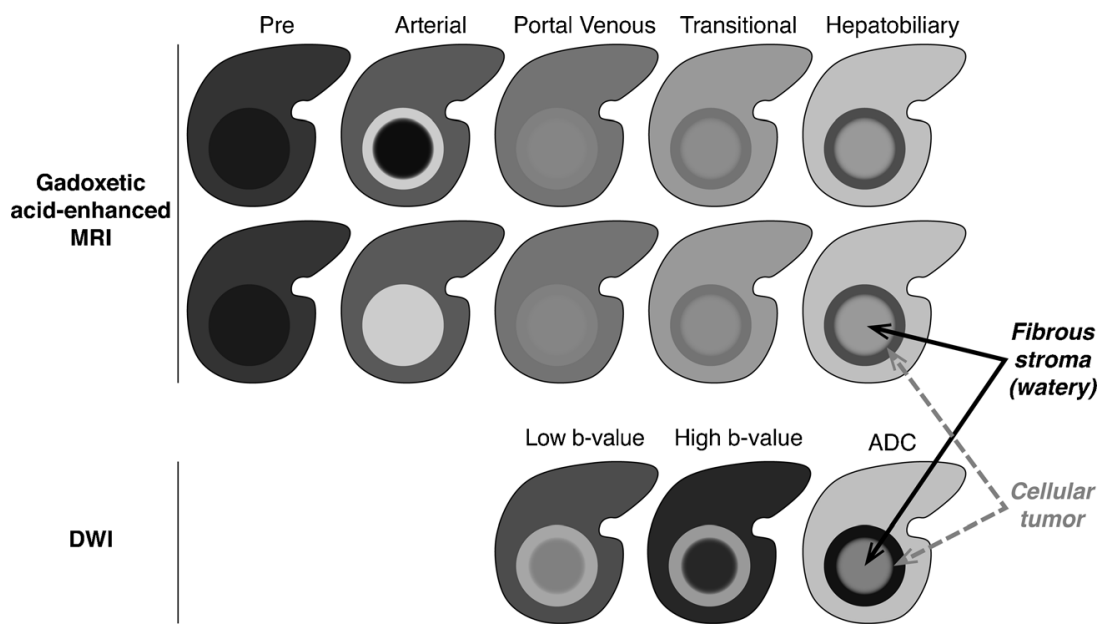

better estimation of liver remnant function, and by accurate detection and staging of tumours.

Consensus statement 3.1 Evidence and experience suggest that gadoxetic acid-enhanced MRI combined with DWI is the most accurate imaging modality for preoperative diagnosis of CRLM. [54/66 (81.8\%) agreement]

Estimation of segmental liver function using MRI, based on hepatic uptake of gadoxetic-acid, has shown good correlation with conventional measures of function, and with disease severity scoring systems [27, 42-44]. Gadoxetic-acidenhanced MRI has shown a high sensitivity and PPV (96\% and 0.91 , respectively) for the pre-operative work-up of patients with CRLM (superior to both MDCT and positron emission tomography [PET]/CT) [45], and was more sensitive than MDCT for the detection of histopathologically proven CRLM, especially those $<1 \mathrm{~cm}$ [46]. Combining gadoxeticacid-enhanced MRI with DWI appears to further improve the detection of CRLM compared with the individual imaging techniques [47, 48], especially for small metastases [49], including patients who have undergone pre-operative chemotherapy [50].

\section{Hepatocellular carcinoma (HCC)}

International guidelines on $\mathrm{HCC}$ management recommend stratification of treatment into potentially curative and palliative therapies according to the severity of underlying liver disease and the number, size and invasiveness of hepatic nodules $[51,52]$.

Consensus statement 3.2 Increasing evidence suggests that gadoxetic acid-enhanced MRI combined with T2-weighted imaging and DWI is an accurate method for the diagnosis and staging of HCC. [63/68 (92.6\%) agreement]

According to the current guidelines, multiphasic MDCT and MRI with extracellular contrast agents are the first-line imaging modalities to characterize lesions in patients at risk for development of HCC. However, features like hyperintensity in DWI and hypointensity in HBP images are considered as valuable contributions to the vascular assessment of such lesions.

Gadoxetic-acid-enhanced MRI has high sensitivity for the diagnosis of HCC, especially those $\leq 2 \mathrm{~cm}$ [34, 53-57], and is more sensitive than MDCT for detecting $\mathrm{HCC} \leq 1 \mathrm{~cm}$ [58]. Pre-operative detection of additional very small liver lesions (potentially leading to recurrence) may change the proposed therapy and increase the chance of curative treatment [59].

DWI increases the detection rate of focal liver lesions compared with breath-hold T2-weighted MRI alone [60]. One recent study showed that on MDCT 64/102 (63\%) small ( $\leq 2 \mathrm{~cm}$ ) HCCs showed a target appearance, while $13(13 \%)$ were not detected [61]. In contrast, 84 (82\%) HCCs demonstrated target arterial hyperintensity, HBP hypointensity and DWI hyperintensity on gadoxetic acid-enhanced MRI, and a further eight hypovascular HCCs could be identified as hyperintense lesions on DWI [61].

\section{Assessment of the liver following non-surgical treatment of liver malignancies}

Locoregional therapies are recommended when surgical resection or transplantation are not options for malignant liver lesions [51, 62, 63]. Subsequent assessment of the liver involves evaluation of therapeutic success, liver injury as a result of the treatment, and detection of recurrence.

Consensus statement 3.3 Gadoxetic acid-enhanced MRI shows promise for assessment of radiation-induced liver injury. [61/67 (91.0\%) agreement]

Reduced gadoxetic-acid uptake (diffuse hypointensity on HBP images) following interstitial brachytherapy correlates with radiation dose-related 
hepatocyte dysfunction [64], and subsequent repair of the parenchyma post-therapy is evident as a reduced area of hypointensity [65]. Thus, gadoxetic-acidenhanced MRI can be used to detect parenchymal damage and assess the value of protective therapies [66]. In addition, the dose range of proton therapy can be similarly assessed [67].

Consensus statement 3.4 Gadoxetic acid-enhanced MRI is useful for follow-up after local and locoregional therapy of $\mathrm{HCC}$ with respect to the assessment of new lesions, but the value of the HBP is questionable for the assessment of local control after thermal ablation in early follow-up. [55/67 (82.1\%) agreement]

Evaluation of sensitivity, specificity and accuracy of unenhanced T1-weighted, T2-weighted and dynamic gadoxetic acid-enhanced MRI to detect HCC recurrence following radiofrequency ablation of the liver showed similar results; addition of HBP images did not improve these results [68]. In many cases, HBP hypointensity due to reactive therapeutic responses around the treated lesion could not be distinguished from tumour recurrence.

\section{Evaluation of liver dysfunction after liver transplant}

Dysfunction in transplanted livers following orthotropic liver transplantation (OLT) may involve structural complications (vascular or biliary complications, HCC recurrence, lymphoproliferative disorder) or more diffuse conditions (acute and chronic rejection, cold ischaemia, recurrent viral hepatitis, autoimmune hepatitis, fibrosis, cirrhosis) $[69,70]$. These conditions are commonly assessed using a combination of serum liver function tests and imaging [70].

Consensus statement 3.5 Preliminary data show a correlation between parenchymal dysfunction and gadoxetic acid uptake and excretion in post-liver transplantation patients. This could play a future role in monitoring OLT dysfunction, and further investigation is warranted. [59/68 (86.8\%) agreement]

Gadoxetic acid-enhanced MRI is a promising tool for regional liver function assessment $[27,42,43]$ and may be a useful, non-invasive, prognostic biomarker for chronic liver graft rejection and patient outcome [71]. Liver transplant recipients with impaired hepatobiliary excretion of gadoxeticacid showed significantly higher median serum bilirubin levels ( 4.9 vs. $1.2 \mathrm{mg} / \mathrm{dL}, P<0.001)$, aspartate aminotransferase ( 89 vs. $41 \mathrm{IU} / \mathrm{L}, P=0.003)$ and alkaline phosphatase (322 vs. $143 \mathrm{IU} / \mathrm{L}, P=0.007)$, and within 1 year, $11 / 20$ patients died or required re-transplantation, while all 31 patients with normal gadoxetic acid excretion survived without retransplantation $(P<0.001)$ [71].
In a recent study [72], a significant correlation was found between relative liver enhancement (RLE) at $20 \mathrm{~min}$ and liver failure in retrospective univariate and multivariate analyses in 73 patients who underwent gadoxetic-acid-enhanced MRI prior to resection of three or more liver segments. Pre-operative RLE was inversely related to the probability of post-operative liver failure [72]. The probability of liver failure was close to zero for a RLE $>100 \%$, suggesting that an RLE of $100 \%$ may be a cut-off to predict patients at risk for liver failure. However, determination of cut-off values must be viewed cautiously and more data is needed to validate these values.

\section{Tips and tricks to optimize imaging with gadoxetic acid-enhanced MRI}

\section{Arterial phase imaging}

Gadoxetic acid differs from extracellular MR-contrast media in that it has higher T1 relaxivity [73], and is formulated at half of the gadolinium concentration with a recommended quarter standard dose $(0.025 \mathrm{~mol} / \mathrm{kg}$ body weight $)$ in half the standard injected volume. Working with a compact bolus can be challenging, but strategies to ensure robust arterial phase imaging are available.

Consensus statement 4.1 Artefacts observed in gadoxetic acid-enhanced MRI in the arterial phase can be reduced by using multiphase pulse sequences with short acquisition times. [58/64 (90.6\%) agreement]

Consensus statement 4.2 Ancillary strategies for reducing arterial phase artefacts include stretching the contrast bolus, sequential $\mathrm{k}$-space filling and patient-tailored timing of the hepatic arterial phase acquisition. [62/64 (96.9\%) agreement]

Artefacts seen in the arterial phase of CE-MRI include breathing or motion artefacts, truncation artefacts and phase ghosting (due to rapid change of gadolinium concentration during k-space acquisition) [74]. Breathing artefacts can be reduced by good patient instructions. Both breathing and truncation artefacts can be addressed by shortening the acquisition time, for example by acquiring multiple short arterial phase sequences rather than a single longer one. In this way, at least one phase will likely coincide with optimal arterial enhancement, and transient artefacts (e.g. due to motion) may contaminate only one of the acquired phases [75]. Truncation or phase-ghosting artefacts can also be reduced by stretching the contrast bolus [76-80]. Furthermore, timing of the contrast bolus can be improved with semiautomated, bolus-triggered techniques. 


\section{Hepatobiliary phase imaging}

\section{Technique optimization}

The signal-to-noise ratio (SNR) of hepatobiliary contrast enhancement is related to the concentration of $\mathrm{CM}$ in the target tissue and the strength of $\mathrm{T} 1$ weighting.

Consensus statement 4.3 For gadoxetic acid-enhanced HBP liver MRI, optimized pulse sequences using high flip angles can improve liver lesion conspicuity and detection. [60/66 (90.1\%) agreement]

Higher flip angles in HBP sequences can increase liver parenchymal contrast enhancement and improve the conspicuity of lesions [81]. At higher flip angles, sensitivity for lesion detection was significantly improved $(89.0 \%$ vs. $79.5 \%$ for small flip angles, $P=0.0003$ ), particularly for small (3$10 \mathrm{~mm})$ lesions $(81.4 \%$ vs. $65.7 \%, P=0.0002)$ and the liver-to-lesion contrast was significantly greater in most large $(\geq 10 \mathrm{~mm}$ ) lesions (378 vs. $150, P<0.05$ ) [82]. The optimal flip angle for imaging in the HBP has been suggested to be around $40^{\circ}[83]$.

\section{Timing of the hepatobiliary phase}

Deciding when to acquire HBP imaging sequences on gadoxetic-acid-enhanced MRI is generally a compromise between shorter and longer delays (patient comfort vs. improved visualization of biliary excretion).

Consensus statement 4.4 The post-injection delay for hepatobiliary imaging with gadoxetic acid is patient and indication specific. While a 20-min delay is acceptable in most cases, a shorter delay may be feasible for parenchymal imaging in some patients. Longer delays may be helpful for biliary imaging. [61/62 (98.4\%) agreement]

Consensus statement 4.5 The post-injection delay for hepatocyte phase imaging with gadoxetic acid is patient and indication specific. Longer delays may be helpful for patients with impaired liver uptake. [53/63 (84.1\%) agreement]

The optimal delay before acquiring HBP images following gadoxetic acid administration was suggested to be $20 \mathrm{~min}$ [84]. Nonetheless, in a variety of patients, adequate HBP images can be obtained well before $20 \mathrm{~min}$ [85-88]. However, HBP enhancement occurs later and more weakly in patients with chronic liver disease, suggesting that a longer delay after injection of gadoxetic acid may be optimal in such patients [85].

For biliary imaging, the signal intensity of gadoxetic acid enhancement in the common bile duct reaches a peak at 30min post-injection in patients with and without chronic liver disease (lower signal intensity in patients with liver disease) [89]. In addition, since gadoxetic acid and bilirubin are taken up by the same family of organic anion transport proteins (OATPs), the presence of an elevated serum bilirubin level is associated with reduced hepatic gadoxetic-acid uptake and poor enhancement in the hepatobiliary phase [54]. For this reason, an elevated bilirubin level is a relative contraindication in some centres for gadoxetic acid, with threshold bilirubin levels from $2.0-5.0 \mathrm{mg} / \mathrm{dL}[54,90]$.

\section{Summary}

Gadoxetic acid-enhanced MRI, including HBP imaging, provides high sensitivity for differentiating FNH and HCA; however, the role of the HBP to aid prediction of HCA subtype requires further research. Specific features of HCC and ICC demonstrated on gadoxetic acid-enhanced MRI can aiding differential diagnosis. Hypointensity in the HBP of gadoxetic acid-enhanced MRI provides high sensitivity for the detection of pre-malignant hepatocellular lesions, although it is currently not possible to differentiate those. Incorporating a combination of ancillary MRI findings can assist in patient risk stratification. Gadoxetic acid-enhanced MRI combined with DWI may be the most accurate imaging modality for the pre-operative diagnosis of CRLM. Gadoxetic acidenhanced MRI also shows promise for evaluating radiation-induced liver injury and the occurrence of new lesions after locoregional therapy, and could play a role in assessing liver dysfunction after transplantation. Various techniques can increase the robustness of gadoxetic acid-enhanced MRI, reduce artefacts and improve lesion conspicuity in HBP images. Post-injection delays for hepatobiliary imaging with gadoxetic acid are patient- and indication-specific.

Acknowledgments The scientific guarantor of this publication is Elmar M. Merkle, MD. The authors of this manuscript declare relationships with the following companies:.

E.M.M. is a member of the Liver Advisory Board and Speaker Bureau for Bayer HealthCare.

C.J.Z. is a member of the Liver Advisory Board, Bayer HealthCare, and the Scientific Cooperation, Bayer HealthCare, and has received honoraria for lectures and travel costs from Bayer HealthCare and Bracco.

C.B. is a member of the Liver Advisory Board, Bayer HealthCare, and has received honoraria for lectures and travel costs from Bayer HealthCare.

M.R.B. is a member of the Liver Advisory Board for Bayer HealthCare, and is Principal Investigator, Investigator-Initiated Study, Bayer HealthCare.

A.H. has been a full-time employee of Siemens AG since 1 June 2004. His function is Associate Director of the Imaging Science Institute Charité, a scientific cooperation between the Charité, University Hospitals of Berlin, Germany and Siemens Healthcare in the form of a privatepublic partnership (PPP). He has received honoraria for lectures and travel costs from Bayer HealthCare.

J.M.L. is a member of the Liver Advisory Board, is Principal Investigator, Investigator-Initiated Study, and has received honoraria for lectures and travel costs from Bayer HealthCare. 
J.R. has received consultancy and lecture fees, funding for investigator-initiated clinical trials, and is a member of the Liver Advisory Board, Bayer HealthCare.

C.B.S. is a member of the Liver Advisory Board and Speaker Bureau for Bayer HealthCare, and is Principal Investigator, Investigator-Initiated Study, Bayer HealthCare.

M-S.Z. is a member of the Liver Advisory Board for Bayer HealthCare, and is Principal Investigator, Investigator-Initiated Study, Bayer HealthCare.

A.B..-S., M.S. and S-L.Y. have no conflict of interest to declare.

This study received funding from Bayer HealthCare. No complex statistical methods were necessary for this paper. Institutional Review Board approval was not required because this is a review paper.

Open AccessThis article is distributed under the terms of the Creative Commons Attribution-NonCommercial 4.0 International License (http:// creativecommons.org/licenses/by-nc/4.0/), which permits any noncommercial use, distribution, and reproduction in any medium, provided you give appropriate credit to the original author(s) and the source, provide a link to the Creative Commons license, and indicate if changes were made.

\section{References}

1. Grieser C, Steffen IG, Seehofer D et al (2013) Histopathologically confirmed focal nodular hyperplasia of the liver: gadoxetic acidenhanced MRI characteristics. Magn Reson Imaging 31:755-760

2. Grazioli L, Bondioni MP, Haradome H et al (2012) Hepatocellular adenoma and focal nodular hyperplasia: value of gadoxetic acidenhanced MR imaging in differential diagnosis. Radiology 262: 520-529

3. Huppertz A, Haraida S, Kraus A et al (2005) Enhancement of focal liver lesions at gadoxetic acid-enhanced MR imaging: correlation with histopathologic findings and spiral CT-initial observations. Radiology 234:468-478

4. Zech CJ, Grazioli L, Breuer J, Reiser MF, Schoenberg SO (2008) Diagnostic performance and description of morphological features of focal nodular hyperplasia in Gd-EOB-DTPA-enhanced liver magnetic resonance imaging: results of a multicenter trial. Investig Radiol 43:504-511

5. Denecke T, Steffen IG, Agarwal S et al (2012) Appearance of hepatocellular adenomas on gadoxetic acid-enhanced MRI. Eur Radiol 22:1769-1775

6. Mohajer K, Frydrychowicz A, Robbins JB, Loeffler AG, Reed TD, Reeder SB (2012) Characterization of hepatic adenoma and focal nodular hyperplasia with gadoxetic acid. J Magn Reson Imaging 36:686-696

7. Bieze M, van den Esschert JW, Nio CY et al (2012) Diagnostic accuracy of MRI in differentiating hepatocellular adenoma from focal nodular hyperplasia: prospective study of the additional value of gadoxetate disodium. AJR Am J Roentgenol 199:26-34

8. Purysko AS, Remer EM, Coppa CP, Obuchowski NA, Schneider E, Veniero JC (2012) Characteristics and distinguishing features of hepatocellular adenoma and focal nodular hyperplasia on gadoxetate disodium-enhanced MRI. AJR Am J Roentgenol 198: 115-123

9. Grazioli L, Olivetti L, Mazza G, Bondioni MP (2013) MR imaging of hepatocellular adenomas and differential diagnosis dilemma. Int J Hepatol 2013:374170

10. Yoneda N, Matsui O, Kitao A et al (2012) Beta-cateninactivated hepatocellular adenoma showing hyperintensity on hepatobiliary-phase gadoxetic-enhanced magnetic resonance imaging and overexpression of OATP8. Jpn J Radiol 30: 777-782

11. Pomfret EA, Washburn K, Wald C et al (2010) Report of a national conference on liver allocation in patients with hepatocellular carcinoma in the United States. Liver Transpl 16:262-278

12. Rizvi S, Gores GJ (2013) Pathogenesis, diagnosis, and management of cholangiocarcinoma. Gastroenterology 145:1215-1229

13. Park HJ, Kim YK, Park MJ, Lee WJ (2013) Small intrahepatic mass-forming cholangiocarcinoma: target sign on diffusionweighted imaging for differentiation from hepatocellular carcinoma. Abdom Imaging 38:793-801

14. Chong YS, Kim YK, Lee MW et al (2012) Differentiating massforming intrahepatic cholangiocarcinoma from atypical hepatocellular carcinoma using gadoxetic acid-enhanced MRI. Clin Radiol 67:766-773

15. Hwang J, Kim YK, Park MJ et al (2012) Differentiating combined hepatocellular and cholangiocarcinoma from mass-forming intrahepatic cholangiocarcinoma using gadoxetic acid-enhanced MRI. J Magn Reson Imaging 36:881-889

16. Jeong HT, Kim MJ, Chung YE, Choi JY, Park YN, Kim KW (2013) Gadoxetate disodium-enhanced MRI of mass-forming intrahepatic cholangiocarcinomas: imaging-histologic correlation. AJR Am J Roentgenol 201:W603-W611

17. Kang Y, Lee JM, Kim SH, Han JK, Choi BI (2012) Intrahepatic mass-forming cholangiocarcinoma: enhancement patterns on gadoxetic acid-enhanced MR images. Radiology 264:751-760

18. Kim SH, Lee CH, Kim BH et al (2012) Typical and atypical imaging findings of intrahepatic cholangiocarcinoma using gadolinium ethoxybenzyl diethylenetriamine pentaacetic acid-enhanced magnetic resonance imaging. J Comput Assist Tomogr 36:704-709

19. Peporte AR, Sommer WH, Nikolaou K, Reiser MF, Zech CJ (2013) Imaging features of intrahepatic cholangiocarcinoma in Gd-EOBDTPA-enhanced MRI. Eur J Radiol 82:e101-e106

20. Chou CT, Chen YL, Wu HK, Chen RC (2011) Characterization of hyperintense nodules on precontrast T1-weighted MRI: utility of gadoxetic acid-enhanced hepatocyte-phase imaging. J Magn Reson Imaging 33:625-632

21. Suh YJ, Kim MJ, Choi JY, Park YN, Park MS, Kim KW (2011) Differentiation of hepatic hyperintense lesions seen on gadoxetic acid-enhanced hepatobiliary phase MRI. AJR Am J Roentgenol 197:W44-W52

22. Bosman FT, Carneiro F, Hruban RH, Theise ND (2010) WHO classification of tumours of the digestive system, 4th edn. International Agency for Research on Cancer (IARC), Lyon

23. Nakashima O, Sugihara S, Kage M, Kojiro M (1995) Pathomorphologic characteristics of small hepatocellular carcinoma: a special reference to small hepatocellular carcinoma with indistinct margins. Hepatology 22:101-105

24. Takayama T, Makuuchi M, Hirohashi S et al (1998) Early hepatocellular carcinoma as an entity with a high rate of surgical cure. Hepatology 28:1241-1246

25. Akai H, Matsuda I, Kiryu S et al (2012) Fate of hypointense lesions on Gd-EOB-DTPA-enhanced magnetic resonance imaging. Eur J Radiol 81:2973-2977

26. Song KD, Kim SH, Lim HK, Jung SH, Sohn I, Kim HS (2015) Subcentimeter hypervascular nodule with typical imaging findings of hepatocellular carcinoma in patients with history of hepatocellular carcinoma: natural course on serial gadoxetic acid-enhanced MRI and diffusion-weighted imaging. Eur Radiol :1-8

27. Sirlin CB, Hussain HK, Jonas E et al (2014) Consensus report from the 6th International forum for liver MRI using gadoxetic acid. J Magn Reson Imaging 40:516-529

28. Kumada T, Toyoda H, Tada T et al (2011) Evolution of hypointense hepatocellular nodules observed only in the hepatobiliary phase of 
gadoxetate disodium-enhanced MRI. AJR Am J Roentgenol 197: $58-63$

29. Motosugi U, Ichikawa T, Sano K et al (2011) Outcome of hypovascular hepatic nodules revealing no gadoxetic acid uptake in patients with chronic liver disease. J Magn Reson Imaging 34: $88-94$

30. Takayama Y, Nishie A, Nakayama T et al (2012) Hypovascular hepatic nodule showing hypointensity in the hepatobiliary phase of gadoxetic acid-enhanced MRI in patients with chronic liver disease: prediction of malignant transformation. Eur J Radiol 81: 3072-3078

31. Kim YK, Lee WJ, Park MJ, Kim SH, Rhim H, Choi D (2012) Hypovascular hypointense nodules on hepatobiliary phase gadoxetic acid-enhanced MR images in patients with cirrhosis: potential of DW imaging in predicting progression to hypervascular HCC. Radiology 265:104-114

32. Joo I, Lee JM, Lee DH, Jeon JH, Han JK, Choi BI (2015) Noninvasive diagnosis of hepatocellular carcinoma on gadoxetic acid-enhanced MRI: can hypointensity on the hepatobiliary phase be used as an alternative to washout? Eur Radiol :1-10

33. Bartolozzi C, Battaglia V, Bargellini I et al (2013) Contrastenhanced magnetic resonance imaging of 102 nodules in cirrhosis: correlation with histological findings on explanted livers. Abdom Imaging 38:290-296

34. Golfieri R, Renzulli M, Lucidi V, Corcioni B, Trevisani F, Bolondi L (2011) Contribution of the hepatobiliary phase of Gd-EOBDTPA-enhanced MRI to Dynamic MRI in the detection of hypovascular small $(</=2 \mathrm{~cm}) \mathrm{HCC}$ in cirrhosis. Eur Radiol 21: $1233-1242$

35. Kogita S, Imai Y, Okada M et al (2010) Gd-EOB-DTPA-enhanced magnetic resonance images of hepatocellular carcinoma: correlation with histological grading and portal blood flow. Eur Radiol 20:2405-2413

36. Sugitani S, Sakamoto M, Ichida T, Genda T, Asakura H, Hirohashi S (1998) Hyperplastic foci reflect the risk of multicentric development of human hepatocellular carcinoma. J Hepatol 28:1045-1053

37. Inoue T, Kudo M, Komuta M et al (2012) Assessment of Gd-EOBDTPA-enhanced MRI for HCC and dysplastic nodules and comparison of detection sensitivity versus MDCT. J Gastroenterol 47: 1036-1047

38. Abdalla EK, Adam R, Bilchik AJ, Jaeck D, Vauthey J-N, Mahvi D (2006) Improving resectability of hepatic colorectal metastases: expert consensus statement. Ann Surg Oncol 13:1271-1280

39. Pawlik TM, Choti MA (2007) Surgical therapy for colorectal metastases to the liver. J Gastrointest Surg 11:1057-1077

40. Leonard GD, Brenner B, Kemeny NE (2005) Neoadjuvant chemotherapy before liver resection for patients with unresectable liver metastases from colorectal carcinoma. J Clin Oncol 23:2038-2048

41. Saif MW (2009) Secondary hepatic resection as a therapeutic goal in advanced colorectal cancer. World J Gastroenterol 15:3855-3864

42. Bae KE, Kim SY, Lee SS et al (2012) Assessment of hepatic function with Gd-EOB-DTPA-enhanced hepatic MRI. Dig Dis 30: $617-622$

43. Katsube T, Okada M, Kumano S et al (2012) Estimation of liver function using $\mathrm{T} 2 *$ mapping on gadolinium ethoxybenzyl diethylenetriamine pentaacetic acid enhanced magnetic resonance imaging. Eur J Radiol 81:1460-1464

44. Yamada A, Hara T, Li F et al (2011) Quantitative evaluation of liver function with use of gadoxetate disodium-enhanced MR imaging. Radiology 260:727-733

45. Chan VO, Das JP, Gerstenmaier JF et al (2012) Diagnostic performance of MDCT, PET/CT and gadoxetic acid (Primovist((R)))-enhanced MRI in patients with colorectal liver metastases being considered for hepatic resection: initial experience in a single centre. Ir J Med Sci 181:499-509
46. Scharitzer M, Ba-Ssalamah A, Ringl H et al (2013) Preoperative evaluation of colorectal liver metastases: comparison between gadoxetic acid-enhanced 3.0-T MRI and contrast-enhanced MDCT with histopathological correlation. Eur Radiol 23: 2187-2196

47. Koh DM, Collins DJ, Wallace T, Chau I, Riddell AM (2012) Combining diffusion-weighted MRI with Gd-EOB-DTPAenhanced MRI improves the detection of colorectal liver metastases. Br J Radiol 85:980-989

48. Tajima T, Akahane M, Takao H et al (2012) Detection of liver metastasis: is diffusion-weighted imaging needed in Gd-EOBDTPA-enhanced MR imaging for evaluation of colorectal liver metastases? Jpn J Radiol 30:648-658

49. Lowenthal D, Zeile M, Lim WY et al (2011) Detection and characterisation of focal liver lesions in colorectal carcinoma patients: comparison of diffusion-weighted and Gd-EOB-DTPA enhanced MR imaging. Eur Radiol 21:832-840

50. Macera A, Lario C, Petracchini M et al (2013) Staging of colorectal liver metastases after preoperative chemotherapy. Diffusionweighted imaging in combination with Gd-EOB-DTPA MRI sequences increases sensitivity and diagnostic accuracy. Eur Radiol 23:739-747

51. (2012) EASL-EORTC clinical practice guidelines: management of hepatocellular carcinoma. J Hepatol 56:908-943

52. Forner A, Llovet JM, Bruix J (2012) Hepatocellular carcinoma. Lancet 379:1245-1255

53. Ahn SS, Kim MJ, Lim JS, Hong HS, Chung YE, Choi JY (2010) Added value of gadoxetic acid-enhanced hepatobiliary phase MR imaging in the diagnosis of hepatocellular carcinoma. Radiology 255:459-466

54. Bashir MR, Gupta RT, Davenport MS et al (2013) Hepatocellular carcinoma in a North American population: does hepatobiliary MR imaging with Gd-EOB-DTPA improve sensitivity and confidence for diagnosis? J Magn Reson Imaging 37:398-406

55. Di Martino M, Marin D, Guerrisi A et al (2010) Intraindividual comparison of gadoxetate disodium-enhanced MR imaging and 64-section multidetector CT in the detection of hepatocellular carcinoma in patients with cirrhosis. Radiology 256:806-816

56. Haradome H, Grazioli L, Tinti R et al (2011) Additional value of gadoxetic acid-DTPA-enhanced hepatobiliary phase MR imaging in the diagnosis of early-stage hepatocellular carcinoma: comparison with dynamic triple-phase multidetector CT imaging. J Magn Reson Imaging 34:69-78

57. Kim SH, Kim SH, Lee J et al (2009) Gadoxetic acid-enhanced MRI versus triple-phase MDCT for the preoperative detection of hepatocellular carcinoma. AJR Am J Roentgenol 192:1675-1681

58. Toyota N, Nakamura Y, Hieda M et al (2013) Diagnostic capability of gadoxetate disodium-enhanced liver MRI for diagnosis of hepatocellular carcinoma: comparison with multi-detector CT. Hiroshima J Med Sci 62:55-61

59. Kudo M, Matsui O, Sakamoto M et al (2013) Role of gadoliniumethoxybenzyl-diethylenetriamine pentaacetic acid-enhanced magnetic resonance imaging in the management of hepatocellular carcinoma: consensus at the Symposium of the 48th Annual Meeting of the Liver Cancer Study Group of Japan. Oncology 84:21-27

60. Parikh T, Drew SJ, Lee VS et al (2008) Focal liver lesion detection and characterization with diffusion-weighted MR imaging: comparison with standard breath-hold T2-weighted imaging. Radiology 246:812-822

61. Park MJ, Kim YK, Lee MH, Lee JH (2013) Validation of diagnostic criteria using gadoxetic acid-enhanced and diffusion-weighted MR imaging for small hepatocellular carcinoma $(<=2.0 \mathrm{~cm})$ in patients with hepatitis-induced liver cirrhosis. Acta Radiol 54:127-136

62. National Comprehensive Cancer Network (2014) NCCN guidelines version 1.2014: colon cancer. NCCN website. Available via 
http://www.nccn.org/professionals/physician_gls/f_guidelines. asp\#colon. Accessed 17 Jan 2014

63. Schmoll HJ, Van CE, Stein A et al (2012) ESMO Consensus Guidelines for management of patients with colon and rectal cancer. A personalized approach to clinical decision making. Ann Oncol 23:2479-2516

64. Seidensticker M, Burak M, Kalinski T et al (2015) Radiationinduced liver damage: correlation of histopathology with hepatobiliary magnetic resonance imaging, a feasibility study. Cardiovasc Intervent Radiol 38:213-221

65. Seidensticker M, Seidensticker R, Mohnike K et al (2011) Quantitative in vivo assessment of radiation injury of the liver using Gd-EOB-DTPA enhanced MRI: tolerance dose of small liver volumes. Radiat Oncol 6:40

66. Seidensticker M, Seidensticker R, Damm R et al (2014) Prospective randomized trial of enoxaparin, pentoxifylline and ursodeoxycholic acid for prevention of radiation-induced liver toxicity. PLoS One 9, e112731

67. Yuan Y, Andronesi OC, Bortfeld TR et al (2013) Feasibility study of in vivo MRI based dosimetric verification of proton end-of-range for liver cancer patients. Radiother Oncol 106:378-382

68. Watanabe H, Kanematsu M, Goshima S et al (2012) Is gadoxetate disodium-enhanced MRI useful for detecting local recurrence of hepatocellular carcinoma after radiofrequency ablation therapy? AJR Am J Roentgenol 198:589-595

69. Ito K, Siegelman ES, Stolpen AH, Mitchell DG (2000) MR imaging of complications after liver transplantation. AJR Am J Roentgenol 175:1145-1149

70. Mehrzad H, Mangat K (2013) The role of interventional radiology in treating complications following liver transplantation. ISRN Hepatol 89:157-164

71. Wibmer A, Aliya Q, Steininger R et al (2012) Liver transplantation: impaired biliary excretion of gadoxate is associated with an inferior 1-year retransplantation-free survival. Investig Radiol 47:353-358

72. Wibmer A, Prusa AM, Nolz R, Gruenberger T, Schindl M, BaSsalamah A (2013) Liver failure after major liver resection: risk assessment by using preoperative Gadoxetic acid-enhanced 3-T MR imaging. Radiology 269:777-786

73. Rohrer M, Bauer H, Mintorovitch J, Requardt M, Weinmann HJ (2005) Comparison of magnetic properties of MRI contrast media solutions at different magnetic field strengths. Investig Radiol 40: $715-724$

74. Erasmus LJ, Hurter D, Naude M, Kritzinger HG, Acho S (2004) A short overview of MRI artefacts. S Afr J Radiol 8:13-17

75. Pietryga JA, Burke LMB, Marin D, Jaffe TA, Bashir MR (2014) Respiratory motion artifact affecting hepatic arterial phase imaging with gadoxetate disodium: examination recovery with a multiple arterial phase acquisition. Radiology 271:426-434

76. Zech CJ, Vos B, Nordell A et al (2009) Vascular enhancement in early dynamic liver MR imaging in an animal model: comparison of two injection regimen and two different doses Gd-EOB-DTPA (gadoxetic acid) with standard Gd-DTPA. Investig Radiol 44: 305-310
77. Haradome H, Grazioli L, Tsunoo M et al (2010) Can MR fluoroscopic triggering technique and slow rate injection provide appropriate arterial phase images with reducing artifacts on gadoxetic acid-DTPA (Gd-EOB-DTPA)-enhanced hepatic MR imaging? J Magn Reson Imaging 32:334-340

78. Schmid-Tannwald C, Herrmann K, Oto A, Panteleon A, Reiser M, Zech C (2012) Optimization of the dynamic, Gd-EOB-DTPAenhanced MRI of the liver: the effect of the injection rate. Acta Radiol 53:961-965

79. Tamada T, Ito K, Yoshida K et al (2011) Comparison of three different injection methods for arterial phase of Gd-EOB-DTPA enhanced MR imaging of the liver. Eur J Radiol 80:e284-e288

80. Tanimoto A, Higuchi N, Ueno A (2012) Reduction of ringing artifacts in the arterial phase of gadoxetic acid-enhanced dynamic MR imaging. Magn Reson Med Sci 11:91-97

81. Bashir MR, Merkle EM (2011) Improved liver lesion conspicuity by increasing the flip angle during hepatocyte phase MR imaging. Eur Radiol 21:291-294

82. Bashir MR, Husarik DB, Ziemlewicz TJ, Gupta RT, Boll DT, Merkle EM (2012) Liver MRI in the hepatocyte phase with gadolinium-EOB-DTPA: does increasing the flip angle improve conspicuity and detection rate of hypointense lesions? J Magn Reson Imaging 35:611-616

83. Nagle SK, Busse RF, Brau AC et al (2012) High resolution navigated three-dimensional $\mathrm{T}(1)$-weighted hepatobiliary MRI using gadoxetic acid optimized for 1.5 Tesla. J Magn Reson Imaging 36:890-899

84. Vogl TJ, Kummel S, Hammerstingl R et al (1996) Liver tumors: comparison of MR imaging with Gd-EOB-DTPA and Gd-DTPA. Radiology 200:59-67

85. Motosugi U, Ichikawa T, Tominaga L et al (2009) Delay before the hepatocyte phase of Gd-EOB-DTPA-enhanced MR imaging: is it possible to shorten the examination time? Eur Radiol 19: 2623-2629

86. Bashir MR, Breault SR, Braun R, Do RK, Nelson RC, Reeder SB (2014) Optimal timing and diagnostic adequacy of hepatocyte phase imaging with gadoxetate-enhanced liver MRI. Acad Radiol 21:726-732

87. Jeong HT, Kim MJ, Park MS et al (2012) Detection of liver metastases using gadoxetic-enhanced dynamic and 10- and 20-minute delayed phase MR imaging. J Magn Reson Imaging 35:635-643

88. Sofue K, Tsurusaki M, Tokue H, Arai Y, Sugimura K (2011) GdEOB-DTPA-enhanced 3.0 T MR imaging: quantitative and qualitative comparison of hepatocyte-phase images obtained $10 \mathrm{~min}$ and $20 \mathrm{~min}$ after injection for the detection of liver metastases from colorectal carcinoma. Eur Radiol 21:2336-2343

89. Takao H, Akai H, Tajima T et al (2011) MR imaging of the biliary tract with Gd-EOB-DTPA: effect of liver function on signal intensity. Eur J Radiol 77:325-329

90. Cruite I, Schroeder M, Merkle EM, Sirlin CB (2010) Gadoxetate disodium-enhanced MRI of the liver: part 2, protocol optimization and lesion appearance in the cirrhotic liver. AJR Am J Roentgenol 195:29-41 